\title{
Itinéraires Itinéraires
}

Littérature, textes, cultures

2014-3 | 2015

Le polar en Europe : réécritures du genre

\section{Corps déviants dans le roman policier français : transgressions et/ou réitérations de normes genrées}

\section{Andrea Hynynen}

\section{OpenEdition}

Journals

Édition électronique

URL : http://journals.openedition.org/itineraires/2597

DOI : $10.4000 /$ itineraires.2597

ISSN : 2427-920X

Éditeur

Pléiade

Référence électronique

Andrea Hynynen, «Corps déviants dans le roman policier français : transgressions et/ou réitérations de normes genrées », Itinéraires [En ligne], 2014-3 | 2015, mis en ligne le 25 septembre 2015, consulté le 19 avril 2019. URL : http://journals.openedition.org/itineraires/2597 ; DOI : 10.4000/itineraires.2597

Ce document a été généré automatiquement le 19 avril 2019

\section{(c) (i) () $\Theta$}

Itinéraires est mis à disposition selon les termes de la licence Creative Commons Attribution - Pas d'Utilisation Commerciale - Pas de Modification 4.0 International. 


\title{
Corps déviants dans le roman policier français : transgressions et/ou réitérations de normes genrées
}

\author{
Andrea Hynynen
}

\section{NOTE DE L'AUTEUR}

L'orthographe trans* sert à signaler une prise de distance vis-à-vis du système binaire du sexe qui oppose le masculin au féminin et refuse toute autre identité de genre (voir Luca Greco 2011 : 64). Nous l'appliquons ici aux personnages dont l'identité de genre n'entre pas dans les catégories femme et homme. Cependant, nous utiliserons ces dernières catégories ainsi que les pronoms personnels correspondants en parlant des personnages dont l'identité de genre s'y prête facilement.

Cet article étudie la représentation de personnages au corps déviant dans un choix de romans policiers écrits par Brigitte Aubert, Virginie Brac, Pierre Lemaitre et Fred Vargas. Tous ont créé des enquêteurs dont l'aspect physique ou le corps invitent à questionner les normes de genre. Les livres étudiés sont Transfixions (1998) et Une âme de trop ${ }^{1}$ (2006) d'Aubert, la trilogie Véra Cabral de $\mathrm{Brac}^{2}$, la trilogie Verhœven de Lemaitre ${ }^{3}$ ainsi que la série Adamsberg de Vargas à partir de Pars vite et reviens tard (2001) ${ }^{4}$. Notre article prend appui sur les études féministes et les études de genre consacrées au roman policier, mais au lieu de se focaliser sur les femmes (auteures et/ou personnages), il se concentre sur le corps déviant et puise dans les théories queer et transgenre ${ }^{5}$. Le corps déviant s'écarte des normes corporelles de diverses manières dans les ouvrages étudiés. Les premières analyses féministes du roman policier américain et britannique datent de la fin des années 1980, suivies par des études de genre ainsi que par des études gaies et lesbiennes et, à un moindre degré, queer ${ }^{6}$. En revanche, le roman policier français reste peu exploré d'un point de vue de genre ${ }^{7}$. Notre article examine l'usage qui est fait des corps déviants 
dans les textes, en analysant si, et de quelles manières, ils sont reliés à l'intrigue. Il vise également à voir dans quelle mesure les corps ne correspondant pas aux normes genrées produisent un effet subversif, ou restent enfermés dans un dispositif hétéronormatif.

Notre corpus témoigne de l'intérêt croissant pour la femme ainsi que pour les minorités sexuelles et de genre manifesté par le genre policier. Il convient de rappeler, suivant Gill Plain (2001: 6), que des transgressions de genre et la sexualité transgressive sont présentes dans le roman policier et le roman noir depuis leurs origines. Toujours est-il que le regard qui y est posé sur ces transgressions se modifie, tout comme le sort accordé aux personnages non conformes. La culture populaire (au sens de popular culture), y compris le roman policier, reflète les changements qui se produisent dans les mentalités et les mœurs en même temps qu'elle contribue à ces évolutions. Il est évident que les représentations du genre et de la sexualité ont considérablement évolué dans le roman policier français, même s'il apparaît que les transformations sont moins marquées et sont arrivées plus tard en France qu'aux États-Unis, en Grande-Bretagne ou dans les pays nordiques ${ }^{8}$. Véronique Desnain (2009: 93-94) compte Aubert, Brac et Vargas parmi les écrivaines françaises qui, à partir de la seconde moitié des années 1990, se sont efforcés de modifier l'image de l'enquêteur du genre policier pour déstabiliser sa tradition masculine hétéronormée, si vivement dénoncée par Maud Tabachnik dans son essai intitulé « Remarques sur la non-place des femmes dans le roman noir» (1997). À notre avis, l'œuvre plus récente de Pierre Lemaitre présente un effort similaire à travers son héros Camille Verhœven.

3 Nous empruntons le terme de « corps déviant » à Jennifer Terry et Jacqueline Urla, qui dans Deviant Bodies (1995), visaient à montrer dans quelle mesure de nombreuses activités scientifiques et artistiques ont été formées par l'idée selon laquelle la déviance sur le plan mental et moral se manifeste dans le corps même. Un tel point de vue postule qu'il existe un lien entre la morphologie du corps et l'esprit déviant. Il était à la base de l'anthropologie criminelle et des méthodes anthropométriques développées à fin du XIX siècle par Alphonse Bertillon et Cesare Lombroso entre autres (Guillo 2008). Linden Peach (2006: 7-10) rappelle que ces théories ont eu une grande influence sur certains livres de Conan Doyle, par exemple, et que l'on peut en trouver des traces encore aujourd'hui. Dans une étude plus récente, centrée sur la culture populaire contemporaine, Niall Richardson (2010) utilise les expressions " corps transgressifs » (transgressive bodies) et « corps horsnorme » (non-normative bodies). Les trois termes - « corps déviant », « corps transgressif » et « corps hors-norme» - sont assez proches pour que nous les traitions ici comme des synonymes; il s'agit de corps qui d'une manière ou d'une autre sortent des normes corporelles imposées par la société. Ces normes sont évidemment plurielles et variables. D'ailleurs, la culture populaire a une grande importance quant à la manière dont de telles normes sont retravaillées et (re)produites (Milestone et Meyer 2012).

Dans notre étude, le corps hors-norme appartient principalement à un enquêteur fictionnel, que celui-ci soit un policier ou un détective amateur, alors que les recherches sur le corps dans le roman policier ont tendance à se concentrer sur le corps des victimes (voir Cingal 2010 ; Peach 2006 : 9-10, 70-80 ; Plain 2001 : 11-13). Dans les études féministes consacrées à la figure de la détective, la vulnérabilité de son corps, sa voix, son regard et sa violence sont accentués, alors que son aspect physique et sa physiologie sont plus rarement abordés, sinon pour dénoncer son hypersexualisation (Walton et Jones 1999: 164 ; Bilat et Haver 2011). Notre étude prend appui sur le lien communément établi entre corps et genre. Quelle que soit la définition donnée du corps, qui pour certains est une 
donnée biologique essentielle et pour d'autres une construction sociale à part entière, pour ne mentionner que les points extrêmes du continuum théorique à ce sujet (Mäntymäki 2004 : 68-88; Milestone et Meyer 2012), le corps est au cœur de la notion de genre. Le corps est «support de discrimination, lieu de différenciation et reflet des préjugés d'une société » (Cingal 2010 : 97). Ainsi, le « spectacle de la corporalité féminine répond à des attentes différentes que celui de la corporalité masculine ", nous rappellent Loïse Bilat et Gianni Haver (2011 : 26).

Il convient à ce propos de noter que, dans notre corpus, la déviance corporelle concerne principalement des personnages jouant un rôle positif dans le récit, soit en tant que héros (sujet actantiel) soit en adjuvant. Sa fonction n'y est pas la même que dans le cas habituel, où le corps déviant appartient au criminel et sert à mettre en relief sa malveillance. Dans les genres policer et noir, la déviance est en effet rattachée principalement au crime et aux criminels, puisque crime et déviance sont étroitement liés (Gregoriou 2009: 1 ; Peach 2006 : 8-12). Beaucoup de criminels et meurtriers mis en scène dans ce type d'ouvrages ont une physionomie ou un physique aberrants. En revanche, le corps hors-norme appartient dans notre corpus au détective, ce qui signifie qu'il se rattache à l'ordre établi, car le détective est traditionnellement une figure autoritaire et le vecteur moral du roman policier ${ }^{9}$. Que le détective fictionnel apparaisse souvent comme un personnage marginal, surtout dans le roman noir, ne change pas cet état de fait ; le détective possède tout de même un statut autoritaire et héroïque. Ainsi, alors que le corps déviant du criminel tend à affirmer les normes corporelles, le corps déviant du détective incite à les remettre en question.

6 En premier lieu, nous aborderons les figures les moins visiblement déviantes parmi celles incluses dans l'étude en analysant le corps déviant du commandant Camille Verhœven, créé par Pierre Lemaitre, et celui de la lieutenante Violette Retancourt qui apparaît dans les romans policiers de Fred Vargas. Dans les deux cas, c'est la taille qui constitue la qualité hors-norme de leur corps: Verhœven ne mesure que 145 centimètres, alors que Retancourt est exceptionnellement grande. Ces personnages et leur corps déviant occupent une place importante dans les romans, mais la déviance corporelle n'y regarde guère que leur portrait respectif. Nous examinerons ensuite des livres où le corps déviant est impliqué dans l'histoire et thématisé un peu plus profondément. La série de Véra Cabral, créée par Virginie Brac, présente un héros intersexe. Née avec un corps présentant des caractéristiques masculines et féminines, Véra assume très jeune le genre féminin mais refuse la correction chirurgicale jusqu'au deuxième livre. Nous analyserons enfin deux livres de Brigitte Aubert offrant une approche différente. Transfixions introduit unE enquêteurE trans* en pleine transition ${ }^{10}$. Le milieu est celui de la prostitution, où figurent plusieurs personnages qui sortent du cadre binaire homme/femme (Hynynen 2013a). Une âme de trop présente un protagoniste trans* qui est à la fois la victime et son propre assassin sans le savoir.

\section{Trop petit ou trop grande par rapport aux normes genrées}

7 Selon Mäntymäki (2004: $112 ; 347-348)$, maints détectives fictionnels possèdent un corps remarquable, à cause de sa hauteur ou de son poids. Une haute taille est fortement valorisante pour les hommes dans le roman policier et sert à affirmer leur masculinité. La taille y est ainsi souvent une caractéristique significative. Pierre Lemaitre et Fred Vargas 
utilisent cet élément pour faire distinguer leur enquêteur du commun, mais au lieu de lui attribuer une taille extraordinaire connotée positivement, ces auteurs optent pour la solution inverse : un corps d'homme trop petit et un corps de femme trop grand. Cela leur permet d'exposer les attentes liées au genre qui sont imposées par la société. Malgré cette analogie, la connotation de ces corps et leur rôle dans l'intrigue diffèrent.

Le tout petit commandant Camille Verhœven est le héros de trois romans policiers écrits par Lemaitre, qui composent la trilogie Verhœven ${ }^{11}$. Le même personnage apparaît également dans deux ouvrages ne faisant pas partie de ladite trilogie: un feuilleton électronique intitulé Les Grands Moyens (2011) et le roman inédit Rosy et John (2013), écrit pour une campagne de promotion de la collection "Le Livre de Poche ${ }^{12}$ ». Le portrait du commandant Verhœven lui accorde deux caractéristiques essentielles, soigneusement annoncées au début de chaque œuvre : « un don inouï pour le dessin » et «l'hypertrophie fotale qui a fait de lui un homme d'un mètre quarante-cinq » (Alex : 22). Cette mesure est consciencieusement répétée tout au long de la série. Le narrateur insiste encore sur la petite taille du commandant en la mettant en contraste avec celle de ses collègues: par exemple, le grand et beau Maleval a « le physique du champion de France junior de judo » (Travail soigné : 47), alors que le divisionnaire Le Guen « fait dans le monumental » (Alex: 22).

9 Les deux qualités de Verhœven proviennent de sa mère, peintre célèbre et fumeuse invétérée. Le corps hors-norme résulte d'une condition médicale due au refus de la mère, elle-même morte d'un cancer, d'arrêter de fumer pendant sa grossesse. Il est d'ores et déjà défini comme une difformité : « un handicap », une " humiliation effroyable » et une «malédiction» (Alex : 22). Le passage contenant ces expressions dévalorisantes est écrit en focalisation interne et trahit ainsi ce que pense Verhœven lui-même. Le narrateur omniscient emploie également le mot "destinée » à ce propos, et révèle que la femme décédée du commandant, Irène, avait réussi à transformer sa taille en " une force ». Il nous paraît toutefois impossible de trouver des indices de la force censée émaner de la taille de Verhœven grâce à l'influence bienfaisante d'Irène, peut-être parce qu'elle meurt à la fin de Travail soigné, ce qui plonge le commandant dans la dépression et alourdit l'atmosphère des romans suivants. La remarque passagère ne semble figurer que pour rappeler au lecteur qu'un handicap peut être transformé en atout. Il est clair que le corps hors-norme est un fardeau dont le protagoniste impute la responsabilité à sa mère. Aussi la relation mère-fils est-elle contaminée par l'amertume; Verhœven aime sa mère en même temps qu'il la méprise, à tel point qu'il projette de vendre toutes ses œuvres dont il a hérité. De fait, le corps déviant n'est présenté que sous une lumière défavorable.

10 Le corps hors-norme de Verhœven caractérise ce personnage, tout d'abord physiquement mais aussi sur le plan mental, puisque la déviance corporelle suscite sans cesse des réactions et des remarques qui influencent le caractère du détective. Le corps représenté est souvent connecté implicitement à des sensations et à des expériences émotionnelles, ainsi qu'à des facultés mentales (Mäntymäki 2004: 111). Confronté aux regards des autres, Verhœven est amené à adopter une contenance méfiante et agressive : « Colérique et mesuré, brutal et manœuvrier, il est assez rare que les gens le comprennent du premier coup " (Alex: 24). Vu sous cet angle, le corps est proposé comme un facteur essentiel quant aux traits de caractère peu flatteurs du héros, sans en être la cause directe. Les excès de colère de Verhœven et sa prouesse ne proviennent pas de la déviance corporelle en soi; ils ont leur origine première dans les attitudes et les réactions des autres. Chez Verhœven, le corps hors-norme se combine avec un sale caractère et un regard 
tranchant, comparé à une lame. Il est particulièrement méfiant à l'égard de tous ceux qui représentent l'autorité, de sorte que Le Guen se demande si c'est «sa taille qui conduit ainsi Camille à s'opposer à l'autorité » (Alex : 321). Évidemment, Camille Verhœven doit aussi faire preuve de compétences professionnelles extraordinaires car sa taille entraverait normalement toute carrière dans la police. À part les remarques passagères au sujet de ses exploits, de sa notoriété et de son «autorité morale » (Travail soigné: 24), les œuvres prêtent peu d'attention à la question de savoir comment Verhœven a atteint sa position. Il est dit à un moment donné qu'il « a trop de talent pour être flic, mas pas assez pour être artiste. Alors, il est flic » (Lemaitre $2013: 22$ ).

11 À part la brève description du héros donnée dès son entrée en scène dans chaque texte, son corps est évoqué dans deux types de situation. Il y est fait référence, d'une part, quand Verhœven rencontre des inconnus (témoins, suspects ou collègues) pour la première fois et, d'autre part, dans des moments d'autoréflexion, où Verhœven commente son propre corps et sa vie. En général, son apparition suscite de l'embarras et de la méfiance. Le regard condescendant des autres est communiqué au moyen de la focalisation interne sur les personnages secondaires qui, dans leurs pensées, traitent Verhœven de "nain » (Alex : 141) ou de "petit qui commande » quand ils ne le disent pas à haute voix. Parfois, son état suscite de la compassion, comme chez ce témoin: "Le handicap de cet homme, c'est comme les animaux abandonnés, ça la transperce [...]. Devant ce spectacle, tout en elle s'apitoie, flageole, défaille, si c'est pas malheureux » (Alex : 37). Ainsi, les livres de Lemaitre mettent en lumière les normes genrées qui conditionnent les attentes sur ce qu'un homme devrait être et signalent combien la conformité corporelle importe pour la position sociale. Il est dit que pour un homme de cette taille, les occasions de rencontrer une femme sont rares (Alex: 49), alors que les chances de se faire insulter ou défier sont d'autant plus nombreuses. Les remarques de ce type suggèrent que l'accomplissement de l'homme se mesure, du moins partiellement, au succès de ses relations amoureuses hétérosexuelles. Il est frappant que ce sous-entendu hétérocentriste ne soit jamais contesté dans le texte. En revanche, la différence sexuelle est accentuée lorsqu'il est déclaré que «Irène était une femme et lui [Verhœeven] un homme. L'infranchissable était là [...]. C'était même grâce à cette distance qu'ils s'étaient rencontrés " (Sacrifices: 106, nous soulignons). Toujours est-il que le corps déviant de Verhœven n'implique que son portrait à lui ; il est anodin tant par rapport à l'enquête que par rapport aux crimes. Dans les œuvres de Lemaitre, la fonction du corps déviant est donc de caractériser le héros dans le but apparent de le singulariser dans la foule des détectives fictionnels classiques et contemporains. Aussi la déviance corporelle est-elle peu présente dans Sacrifices, le dernier livre, sans doute parce que le lecteur est censé connaître le protagoniste.

Contrairement à Verhœven, Violette Retancourt est un personnage secondaire, adjuvant du principal héros vargassien, le commandant Jean-Baptiste Adamsberg. Ces deux personnages ont pourtant plusieurs points en commun quant à leur corps hors mesure et quant aux effets qu'il produit. Retancourt se remarque par sa taille massive et ses compétences polyvalentes: "un mètre soixante-dix-neuf et cent dix kilos, aussi intelligente que puissante, et capable [...] de convertir son énergie à sa guise » (Sous les vents... : 20). C'est une femme trop grande et trop forte. Personnage mineur dans Pars vite et reviens tard (2001), elle est mise en avant dans Sous les vents de Neptune (2004) et s'impose vite comme le "plus puissant lieutenant» dans la brigade d'Adamsberg (Un lieu... : 49). Retancourt réapparaît dans tous les livres subséquents, mais après lui avoir accordé un 
rôle capital dans Sous les vents de Neptune et Dans les bois éternels (2006), Vargas la délaisse un peu dans ses deux derniers romans : Un lieu incertain (2008) et L'Armée furieuse (2011).

Retancourt est présentée comme une "déesse toute-puissante " (Sous les vents... : 36), louée par des personnages avec qui le lecteur est invité à sympathiser, y compris le protagoniste Adamsberg et ses adjoints principaux Danglard et Veyrenc (Hynynen 2013b). En revanche, des collègues machistes, aux yeux desquels une aussi grosse femme est soit laide soit invisible, se moquent d'elle sans gêne. C'est « une erreur de la nature », dit le lieutenant Noël (Dans les Bois... : 91). La taille et le poids de Retancourt empêchent les gens de voir sa beauté, reconnue seulement par quelques rares personnages dont le jugement n'est pas conditionné par les normes culturelles : « Elle est très belle, dit Zerk. Adamsberg regarda son fils d'un air étonné, car la beauté n'était sûrement pas le caractère premier de Violette Retancourt. Ni la grâce, ni la nuance, ni l'amabilité » (L'Armée furieuse : 39). Ce passage résume d'ailleurs parfaitement ce que la féminité est censée être : beauté, grâce, nuance et douceur. Adamsberg a beau défendre vigoureusement sa lieutenante, il pense lui-même qu'elle "n'était pas exactement une femme au sens convenu du terme " (Un lieu...: 93). Tout comme Lemaitre, Vargas établit une opposition entre, d'un côté, les collègues qui savent regarder au-delà de l'apparence physique et qui reconnaissent la valeur du détective au corps hors-norme, et, de l'autre côté, ceux dont le jugement négatif est fixé par la première impression visuelle. À l'instar de Verhœven, Retancourt atteste qu'un corps hors-norme entrave sérieusement la vie amoureuse et sexuelle. Il est indiqué à plusieurs reprises qu'on la présume vierge du fait que son corps sort de la norme féminine. Le brigadier Favre annonce avec moquerie "la défloration de la Violette » quand Adamsberg dit avoir besoin d'elle (Sous les vents... : 44), tandis que le commandant lui-même s'inquiète pour elle lorsqu'ils traquent une meurtrière en quête de femmes vierges (Dans les bois... : 355). Contrairement à Verhœven qui regrette cette condition, Retancourt opte pour l'abstinence et l'indifférence.

Le corps hors-norme de Retancourt sert avant tout à la mettre à part. Étant donné que les personnages insolites ou bizarres sont la marque distinctive des romans policiers de Vargas (Sudret 2010), il n'est guère étonnant d'y trouver un personnage comme Retancourt. Pourtant, son corps hors-norme joue parfois un rôle dans le cours des événements. Quand Adamsberg est à tort accusé d'un meurtre au Canada, Retancourt l'aide à fuir la police canadienne pour qu'il puisse entreprendre une contre-enquête et prouver son innocence. Elle cache Adamsberg collé à son dos, sous sa robe de chambre, pendant que les agents de police fouillent la chambre. Grâce à sa taille et à sa force, elle parvient à porter le poids d'Adamsberg et à le garder ainsi caché le temps qu'il faut (Sous les vents... : 268). Sa puissance surnaturelle lui permet également de survivre, contre toute attente, lorsqu'elle se fait enlever et empoisonner par la meurtrière de Dans les bois éternels. Qu'elle survive et rejoigne l'équipe est essentiel pour que l'enquête aboutisse. Ce corps hors-norme et la force qui en découle s'avèrent donc aussi utiles pour les enquêtes que ses compétences professionnelles.

15 Vargas insiste sur le lien entre normes corporelles et genre, dans la mesure où les insultes adressées à Retancourt, en raison de son grand corps, portent quasiment toujours sur son manque de féminité. Chez Lemaitre, l'association établie entre la taille du corps et la masculinité est moins explicite. De plus, Retancourt se fait défendre et louer tant par Adamsberg que par d'autres personnages, même si elle n'en a guère besoin, ce qui peut se lire comme une prise de position contre le sexisme. De fait, son corps hors-norme est le plus souvent décrit en des termes positifs, tant par le narrateur que par d'autres 
personnages : grand, puissant, divin, etc. Ses détracteurs sont par contre sévèrement critiqués : par exemple Noël, dont il fut question plus haut, est «brutal », « vulgaire » et «primitif» (Un lieu...: 86). L'univers vargassien contient en outre d'autres personnages qui sont visiblement censés jouer avec les normes genrées, parmi lesquels la musicienneplombière Camille Forestier et l'historien-femme de ménage Marc Vandoosler. En revanche, même ceux qui défendent Verhœven n'abordent jamais la question de son corps. Ils le soutiennent par amitié et à cause de sa compétence professionnelle. Par conséquent, les opinions discriminatoires suscitées par la déviance corporelle ne sont pas remises en question, surtout que Verhœven, qui «ne s'aime pas beaucoup » (Alex : 24), semble avoir lui-même intégré une attitude condescendante et honteuse.

\section{Le corps hors-norme dissimulé}

16 Véra Cabral, personnage principal et narratrice autodiégétique dans trois romans policiers écrits par Virginie $\mathrm{Brac}^{13}$, est psychiatre au Centre d'interventions psychiatriques de Paris. Son travail consiste à intervenir dans des situations d'urgence nécessitant une expertise psychiatrique. Ce faisant, elle découvre par hasard divers crimes qu'elle contribue à résoudre. Il s'agit donc d'un détective amateur.

Véra Cabral est intersexe. Son corps ne rentre pas dans l'opposition binaire corps masculin / corps féminin, mais, ayant choisi le genre féminin, elle vit en femme. Ceci est possible parce qu'elle a "un handicap discret», comme elle le dit (Tropique... : 207). Le personnage est dès le début de la trilogie présenté au lecteur comme une femme au moyen du genre grammatical et de son prénom: "J'étais une psy très comme il faut "; "Après tout, personne ne m'a braqué un pistolet sur la tempe en ordonnant: "Véra, arrête le cabinet" " (Tropique... : 7, 5, nous soulignons). De même, tous les personnages secondaires traitent systématiquement Véra comme si elle était une femme à part entière. Nous respecterons ici le choix linguistique fait par Brac, tout en reconnaissant que cela pourrait être pris pour un biais hétéronormatif. Il ne faut pas oublier que, pour le grand public, la question de savoir comment outrepasser le genre grammatical n'avait guère lieu de se poser au moment où le livre fut publié. Surtout, grâce au respect des règles du genre grammatical (féminin ou masculin), Brac peut créer un mystère autour du corps déviant. Un usage linguistique hors du commun serait trop révélateur.

Le lecteur ignore d'abord l'état intersexe de Véra, même si Tropique du pervers contient de nombreux indices invitant à se poser des questions sur son identité et sa personnalité, à commencer par la phrase inaugurale: «j'ai un faible pour les dingues» (Tropique... : 7). Des remarques peu précises parsèment le texte: «le choc, l'horreur du jour de l'accouchement quand on lui a présenté son bébé. Ce bébé, c'était moi » et « Il n'y en a pas deux comme moi » (Tropique... : 21, 41). Les allusions répétées à la chirurgie ainsi que le fait que personne, à part sa mère, n'a jamais vu le corps nu de Véra suggèrent que «ce truc » secret (Tropique... : 59) a quelque chose à voir avec son corps. La narratrice évite pourtant toute description détaillée du corps hors-norme, qui n'est montré ni aux autres personnages ni au lecteur. Ainsi, ce roman utilise les stratégies classiques appartenant à la formule du roman policier - indices et fausses pistes - pour augmenter la tension, et crée une énigme secondaire en parallèle à l'enquête criminelle, notamment celle du corps dissimulé du protagoniste. Même après avoir révélé au lecteur qu'elle est intersexe et que l'intervention chirurgicale, proposée par son médecin et ses sœurs, consisterait à corriger les parties génitales pour lui donner le corps d'une femme - une révélation qui arrive au 
milieu de Tropique du pervers -, Véra continue à cacher ce fait à son entourage. Selon Faye Stewart, ceci est une stratégie typique du roman policier queer où le mystère entourant l'identité sexuelle ou de genre de certains personnages se mêle à l'enquête visant à découvrir l'identité du coupable. Dans les deux cas, le lecteur essaie de déchiffrer la vérité à partir de bribes d'informations. Stewart $(2014: 6)$ suggère que, dans un récit queer, la sexualité et le genre sont énigmatiques de manière à insister sur la nécessité d'interpréter et de déchiffrer les identités. Pourtant, pour Stewart le roman policier queer rejette l'amour heureux et romantique qui est, en revanche, très important dans le roman policier lesbien, à tel point qu'il prend le pas sur l'enquête policière (Ibid.: 12). Quant à la série Véra Cabral, elle perd graduellement ce côté queer et évolue vers un modèle bien plus conformiste.

Étant donné que le secret du corps déviant est dévoilé dans le premier livre de la série, les deux suivants sont organisés autrement. Néanmoins, Brac reste fidèle à son style et continue à introduire un récit secondaire centré sur la vie privée de Véra afin de créer du suspense et de titiller la curiosité du lecteur. Dans Notre-Dame des barjots, Véra revoit le docteur Hugo Markovitch dont elle était amoureuse quand elle faisait ses études. Sa présence met Véra mal à l'aise parce que leur dernière rencontre remonte à un incident traumatisant, au cours duquel Markovitch est venu à son secours. Hantée par cette expérience qui l'a fait changer de formation, Véra n'arrête pas de se demander s'il s'en souvient encore, s'il avait vu, sans pour autant expliquer ce qui s'était passé et ce que Markovitch aurait pu voir. La narratrice fait allusion à cet incident mystérieux à plusieurs reprises (voir Notre-Dame... : 40, 70, 103, 191, 223), mais il n'est éclairé que vers la fin du livre lorsque Véra explique :

Cette nuit-là, dans la salle de garde, des internes déchainés avaient foutu une fille à poil, la bâillonnant à l'élastoplast pour l'empêcher de crier. Sauf que ce n'était pas exactement une fille, ni tout à fait un garçon, et ils s'étaient tus, médusés. C'est à ce moment-là qu'Hugo est entré... (Notre-Dame... : 257).

Il s'avère que Markovitch avait bien vu son corps hors-norme, raison pour laquelle il est attiré par Véra, car «les femmes [l']ennuient]» et il trouve que toutes les relations hétérosexuelles sont pareilles (Notre-Dame... : 293). Ceci est un parfait exemple de ce que Richardson (2010: 211) nomme l'« érotisation du corps transgressif». La culture populaire a tendance à désexualiser le corps transgressif pour le rendre moins offensif - comme Vargas le fait avec Retancourt-, mais parfois celui-ci est, au contraire, hypersexualisé. En revanche, Double Peine s'attache peu à la déviance corporelle. Véra qui s'est fait opérer dans le livre précédent vit ici en couple avec Hugo, mais leur amour est troublé par l'infidélité d'Hugo ainsi que par l'apparition d'un mystérieux policier algérien, dont Véra tombe amoureuse. Ici, le récit secondaire se construit à partir de la vie amoureuse de Véra et puise dans les clichés sentimentaux, y compris le coup de foudre, le jeu de la séduction, la jalousie et l'obstacle à l'amour. Alors que l'on trouve encore dans Notre-Dame des barjots $(95,257,296)$ des remarques passagères portant sur l'opération corrective qui n'a pas vraiment changé la vie de Véra, Double peine n'en parle plus guère.

Outre ces récits secondaires, le corps hors-norme de Cabral compte dans le récit central du crime et de l'enquête, du moins dans Tropique du pervers. L'héroïne y découvre qu'un cercle de sadomasochistes très puissant est responsable de plusieurs meurtres survenus lors de leurs séances de torture qui sont filmées pour de riches clients. S'étant emparée de son dossier médical, la secte enlève Véra et projette de la tuer en lui coupant les parties génitales dans une opération diffusée en direct sur un site web secret. L'auteur se 
sert ainsi du corps hors-norme du détective pour exposer les goûts pervers des criminels dans ce roman, dont la déviance et les perversions sexuelles sont les thèmes principaux. La mise en scène de cette secte et de ses activités transgressives et nocives, diminue la charge potentiellement offensive du corps déviant de Cabral. À côté de ces adversaires meurtriers et pervertis, Véra Cabral en devient d'autant plus sympathique aux yeux du lecteur. Les livres suivants traitent d'autres thématiques, à savoir la corruption dans le monde scientifique et médical, le racisme et le traumatisme issu de la guerre d'Algérie, faisant passer la déviance corporelle au second plan.

\section{Suspect, victime et enquêteur trans*}

22 Le personnage transsexuel est, dans le roman policier, le plus souvent un criminel. Le tueur en série transsexuel psychopathologique est de nos jours une figure familière, même si les protagonistes trans* se répandent depuis quelques années dans la culture populaire, surtout dans les séries télévisées et les cinémas américain et britannique (Thomas et Espineira 2015; Halberstam 2005; Richardson 2010). Une approche intéressante à ce sujet nous est offerte par Brigitte Aubert. Paru en 1998, son livre Transfixions introduit un personnage principal trans*, qui enquête sur plusieurs meurtres de prostituées, dont la police l'accuse. Écrit à la première personne, le récit épouse le point de vue de Bo', né Beaudoin Ancelin, transsexuel MF et masochiste. Sa transsexualité est vite annoncée, et le corps hors-norme exposé, contrairement à ce qui se passe avec Véra Cabral. Le personnage est à la fois détective amateur, suspect et victime, car il se fait enlever et presque tuer par l'assassin. À la différence des détectives déjà abordés, ce n'est pas une figure sérielle. Même si le livre d'Aubert n'est plus très récent, il était plutôt exceptionnel pour son temps et nous propose un exemple intéressant de la manière de traiter des corps déviants dans un récit policier.

Dans le cas de Bo', il ne s'agit pas seulement du fait que le corps ne correspond pas au genre ; le personnage a commencé, puis interrompu, le traitement hormonal et l'épilation électrique, et il se trouve à présent dans l'entre-deux. Son visage illustre cet état corporel hors-norme : « une moitié du visage [est] imberbe et l'autre velu » (Transfixions : 69). Bo' maîtrise le passing et sait se présenter soit en jeune femme soit en garçon selon les circonstances, mais son but est de se faire opérer et de devenir une femme « qui n'en [a] pas que l'air» (Transfixions: 16). La transsexualité de Bo' se réfère donc au modèle classique «d'un sexe à l'autre» (Foerster 2012: 189), ce que confirme la métaphore suivante du train allant d'un point de départ à un point d'arrivée nettement séparés : « Ici on voyage en capsule de chair, de la planète homo erectus à la planète bella donna " ( Transfixions : 18). Selon Richardson (2010:156), le corps transsexuel pré-opération, auquel correspond le présent état transitionnel de Bo', défie toutefois la binarité de genre tant que la transition reste inachevée. L'échange suivant de paroles entre Bo' et Stéphanie au sujet de Johnny illustre combien la définition du genre devient difficile dans de tels cas :

- Il n'aime pas les hommes.

- Justement, tu [Bo'] n'est pas vraiment un homme.

- Justement, j'en suis vraiment un, si tu vois ce que je veux dire.

- Laisse tomber, conclut-elle [Stéphanie]. (Transfixions : 155)

L'enquêteurE n'est pas seulE à posséder un corps déviant dans ce roman. Bo' fréquente d'autres personnages trans* qui se trouvent à différents stades du processus de transition d'un sexe à l'autre. Certains aspirent à un changement de sexe total avec traitements 
chirurgicaux et hormonaux, alors que d'autres préfèrent rester à l'entre-deux ou optent pour une apparence de genre opposée à leur sexe sans modifier le corps. Le roman prête également attention aux facteurs d'ordre pratique qui peuvent entraver le processus, tels que le manque d'argent ou de soutien familial et la méfiance manifeste dans les professions médicale et psychiatrique. L'« opération " fait l'objet de débats et suscite parfois des doutes, même si elle est le but visé par la plupart: "Écoute Steph, tu t'es jamais demandé ce que tu feras une fois opéré ? Parce que tous les mecs avec qui tu couches, ce qu'ils veulent c'est une femme en apparence, mais un garçon au lit» ( Transfixions : 155). Il est en outre fait mention de la pratique de modifications corporelles ainsi que de travestis qui ne font ceci que pour leur travail dans la prostitution. Ces multiples expressions de genre et d'infractions aux normes corporelles donnent lieu à une utilisation complexe du genre grammatical dans le texte (Hynynen 2013a). Par exemple, le pronom personnel féminin «elle» renvoie à Stéphanie (Steph) dans le premier des deux passages que nous venons de citer, alors que le participe passé « opéré » utilisé dans la deuxième citation dit que ce même personnage est masculin. Tout le récit étant narré par Bo', cette variation ne s'explique pas par une différence de langage entre le narrateur et le personnage.

Le corps déviant est un élément important dans l'intrigue tant par rapport aux crimes que par rapport à l'enquête. Il a déjà été constaté que Bo' se fait kidnapper par le meurtrier. Cela arrive parce que ce dernier, le sadique Johnny, est l'amour impossible de Bo', qui n'arrive pas à y renoncer et continue à poursuivre Johnny quand celui-ci veut disparaître après les meurtres. Homosexuel refoulé, Johnny transforme son désir inavouable pour Bo' en rage et veut le tuer. En outre, la question du corps hors-norme intervient dans le motif du meurtrier qui ne veut tuer que des prostituées qui sont des femmes, et s'arrête au milieu de l'acte quand il tombe sur un travesti. Cet incident permet à l'enquêteurE de mieux comprendre le mobile des meurtres et de diriger l'enquête. Le corps hors-norme de la victime qui survit conditionne ainsi les événements à venir.

Aubert retourne au thème trans* dans Une âme de trop, publié en 2006. Ce roman est composé de douze chapitres écrits à la première personne, avec pour narratrice l'infirmière Elvira, suivis d'un treizième rapporté par un narrateur omniscient et d'un épilogue. Les chapitres alternent avec douze « incisions » qui sont des bribes de discours décousus tenus par une personne anonyme qui parle de violer et de massacrer des femmes : «Jambes écartées, cuisses écartées, [...] ventre éventré, il faut savoir trancher au vif du sujet » (Une Âme... : 56). Le sexe de ce narrateur anonyme n'est pas déclaré, mais les paroles laissent entendre que c'est un homme. À première vue, ce roman ne contient pas de personnages dont le corps sort de la norme; nous sommes bien en face de femmes et d'hommes à l'apparence ordinaire et d'un meurtrier inconnu recherché.

Le lecteur comprend peu à peu que les « incisions » sont les paroles du meurtrier qui a assassiné plusieurs femmes ressemblant à Elvira. Cette dernière s'enferme dans l'appartement qu'elle loue à Steven, un collègue de l'hôpital qui vit en dessous de chez elle, et qui est propriétaire de la maison. Troublée par les meurtres, Elvira essaie sans succès de prévenir l'inspecteur Alvarez de ses soupçons et ses peurs, qu'elle transmet également au lecteur. Le roman suit un modèle de thriller assez standard avec une énigme à résoudre - l'identité du meurtrier - et une tension grandissante due au fait que le protagoniste risque d'être la victime suivante. Cependant les indications transmises par Elvira sont un peu douteuses du fait de son instabilité psychologique. Il lui arrive des 
choses étranges, des objets disparaissent ou sont déplacés, comme si quelqu'un entrait chez elle à son insu. Enfin, la révélation qui clôt le récit fournit un élément de surprise.

Vers la fin, Elvira entre dans l'appartement de Steven pour le prévenir du danger et y trouve trois cadavres. Au moment où elle comprend qu'elle s'est trompée, Steven apparaît : " Nous sommes là, côte à côte, il me regarde en souriant, une main derrière le dos. Je sais ce qu'il y a dans cette main. Un scalpel bien affûté » (Une Âme... : 273). La scène se termine au moment où Elvira décrit les coups de couteau donnés par Steven et raconte sa propre mort. L'incident est raconté de manière à établir la présence de deux personnages : la victime, Elvira, y voit et décrit son meurtrier, Steven. Pourtant, ces deux personnes ne sont qu'une : l'inspecteur Alvarez arrive trop tard sur la scène du crime et $y$ trouve Steven Rosetti, alias Elvira, «[qui] s'est tranché la gorge », par haine de soi (Une Âme... : 280). Celui-ci est un "transformiste corps et âme ", devenu meurtrier parce qu'il ne supportait pas son côté féminin et voulait tuer les femmes qui y ressemblaient (Une Âme... : 281). Ici, ce n'est pas tant le corps qui est déviant que l'esprit, divisé en deux parties qui s'ignorent mutuellement. Il n'empêche que ce roman policier renoue avec la figure du tueur en série psychopathe dont les actes sont motivés par une identité sexuelle troublée. On ne peut s'empêcher de penser au film Psychose d'Alfred Hitchcock, quand il est fait allusion à la mère de Steven, morte juste avant l'arrivée d'Elvira dans la maison. Il n'est pas anodin que Steven, qui est un homme biologique, haïsse sa partie féminine, en d'autres mots son côté transsexuel. Toute l'intrigue repose sur l'ignorance du lecteur face à ce transsexualisme non avoué, ignorance nécessaire pour qu'il y ait du suspense et de la peur ainsi qu'une énigme et un dévoilement final.

\section{Le corps déviant de l'enquêteur et les normes de genre}

Ce qui précède donne quelques exemples des multiples manières dont on peut traiter la déviance corporelle de l'enquêteur dans un roman policier. Le corps hors-norme est parfois une caractéristique personnelle isolée, assez insignifiante au vu de la totalité de l'œuvre et des valeurs qu'elle communique. En même temps, ce corps est susceptible de susciter un débat autour de normes corporelles et de genre, ou bien, il peut avoir une fonction très précise dans la construction de l'énigme, source de tension et/ou de curiosité.

La question de la signification du corps déviant par rapport aux normes (genrées) est peut-être plus délicate quand celui-ci n'est que la marque distinctive d'un seul personnage placé dans un milieu parfaitement hétérocentriste, comme chez Vargas et Lemaitre. La petite taille de Verhœven influence, certes, son comportement et ses relations interpersonnelles mais pas sa compétence professionnelle ni les enquêtes qu'il mène. Quant à Retancourt, sa force et sa grandeur sont importantes pour le cours des événements, mais il s'agit de péripéties secondaires qui auraient pu être arrangées autrement sans que la progression du récit en souffre trop. Étant donné que Retancourt se soumet à son destin de femme laide inapte à rencontrer l'amour ou le plaisir, ses quelques remarques laconiques sur les effets inévitables de la laideur féminine n'ont pour effet que de reproduire ces mêmes normes incontestées. Sa placidité peut, évidemment, aussi se lire comme une prise de distance vis-à-vis du lieu commun qui veut que le couple et l'amour soient la chose la plus importante dans la vie d'une femme. La détective du roman policier féministe est, de fait, très souvent célibataire ou divorcée, car cela permet d'insister sur sa compétence et son indépendance (Munt 1994 : 17-19, 40-45, 197, passim). 
De ce point de vue, la vie célibataire de Retancourt n'a rien d'un échec, tandis que pour Verhœven c'en est un. Il n'empêche que, tant que les romans vargassiens continuent d'associer la féminité accomplie à la beauté, à la sensualité et à la douceur, ils ne déstabilisent guère le genre (Hynynen 2013b). Verhœven et Retancourt troublent peu les normes masculine et féminine, même si les livres révèlent combien l'apparence physique de la personne, tout particulièrement la morphologie corporelle, importe quant à la manière dont son identité de genre est perçue par les autres, peut-être même par la personne elle-même. En fin de compte, ces deux enquêteurs restent ancrés dans le système binaire masculin-féminin dans un univers entièrement hétéronormé, malgré le fait qu'un aspect de leur être ne correspond pas parfaitement aux critères standards de la féminité ou de la masculinité. La fonction de ces détectives est plutôt d'occuper la position de l'exclu, à l'encontre duquel la norme se définit.

31 Dans le premier livre de Virginie Brac, le lecteur découvre petit à petit que le secret de Véra Cabral est son corps intersexe non opéré. Cela donne lieu à des réflexions sur la chirurgie corrective et sur la nécessité de choisir un genre. Tropique du pervers révèle ce que sont l'intersexe et ses modes de traitement. Le livre s'intéresse aussi à des pratiques sexuelles minoritaires : l'homosexualité et le sadomasochisme, dont l'une est soutenue et l'autre condamnée. Toutefois, Véra s'est fait opérer dans le deuxième livre, dans lequel ses soucis amoureux prennent le relais. Toute mise en question de l'hétéronormativité que les deux livres précédents pourraient susciter est effacée du troisième. Ainsi, ce personnage suit un parcours normalisant qui consiste à exorciser le corps déviant et rétablir le système binaire du sexe, où le genre et le corps sont en conformité. Que les livres imposent une image hétérocentriste de l'amour en couple, déclarée par Véra qui dit, lors de son premier baiser, que c'est la première fois qu'elle se sent « une femme, une vraie " (Tropique...: 172), affaiblit aussi leur force subversive, d'autant plus que l'importance du récit d'amour augmente au fur et à mesure.

Dans Transfixion, le corps, ou plutôt les corps hors-norme, conditionnent l'histoire de sorte que le binarisme masculin/féminin est profondément remis en question. La thématique du genre est mise en avant non seulement à travers le portrait du héros autodiégétique, mais aussi au moyen de ses amis et du crime, car le meurtrier, un homosexuel refoulé, ne tue que des prostituées, ce qui a de l'importance pour l'enquête. C'est là le livre du corpus où le questionnement des normes corporelles, sexuelles et genrées est le plus appuyé. En revanche, les artifices du roman policier priment sur la thématique des normes corporelles et genrées dans l'autre roman d'Aubert, Une âme de trop. Que le protagoniste soit un homme qui se travestit en femme, sans s'en apercevoir, sert uniquement à faire progresser l'intrigue et à dérouter le lecteur, d'autant plus que ce travestissement est expliqué par une psychose. Il n'empêche qu'Aubert a construit son récit de manière à manipuler habilement le lecteur pour produire les effets souhaités. Une âme de trop est un thriller réussi, mais son intrigue interdit d'approfondir la discussion du transsexualisme. Ce livre révèle qu'il y a parfois une tension très forte par rapport à la subversion du genre entre la formule du roman policier et le contenu thématique, mais cela ne s'applique pas à tous les livres.

Cette étude montre, d'un côté, que le roman policier se prête parfaitement au projet de problématiser et de retravailler les questions de genre et de sexualité et expose la visibilité grandissante de personnages non conformes. De l'autre côté, elle révèle que les romans les plus récents ne sont pas forcément les moins hétéronormés, de même qu'une œuvre ou une série initialement subversives peuvent être ajustées et normalisées avec le 
temps. Pour conclure, nous constatons aussi que si une meilleure visibilité et la médiatisation grandissante des minorités sexuelles et de genre contribuent à promouvoir la tolérance et à augmenter la compréhension auprès du grand public, elles suscitent aussi des questions importantes à propos de l'appropriation de ces groupes par la majorité, d'autant plus lorsqu'ils sont transformés en spectacles divertissants à des fins commerciales (Thomas et Espineira 2015; Stewart 2014: 196, note 10). Cette piste reste cependant à explorer dans des recherches subséquentes.

\section{BIBLIOGRAPHIE}

Barfoot, Nicola, 2007, Frauenkrimi/polar féminin: Generic Expectations and the Reception of Recent French and German Crime Novels by Women, Francfort, Peter Lang.

Bilat, Loïse et Haver, Gianni (dir.), 2011, Le héros était une femme : le genre de l'aventure, Lausanne, Antipode.

Charest, Danielle, 2006, Crimes suspects : femmes et hommes dans le roman policier, Paris, Pepper.

Cingal, Delphine, 2010, «Lectures du corps : de Sherlock Holmes à Kay Scarpetta ", dans

G. Menegaldo et M. Petit (dir.), Manières de noir. La fiction policière contemporaine, Rennes, PUR, p. 96-111.

Desnain, Véronique, 2009, «Gender and Genre: Women in French Crime Writing », dans

C. Gorrara (dir.), French Crime Fiction, Cardiff, University of Wales Press, p. 86-106.

Foerster, Maxime, 2012, Elle ou lui ? Une histoire des transsexuels en France, Paris, La Musardine.

Greco, Luca, 2011, « Un soi pluriel : la présentation de soi dans les ateliers Drag King », dans N. Chetcuti et L. Greco (dir.), La Face cachée du genre, Paris, Presses Sorbonne Nouvelle, p. 63-83.

Gregoriou, Christiana, 2009, Deviance in Contemporary Crime Fiction, Basingstoke, Palgrave Macmillan.

Guillo, Dominique, 2008, « Bertillon, l'anthropologie criminelle et l'histoire naturelle : des réponses au brouillage des identités », Crime, Histoire \& Sociétés / Crime, History \& Societies, vol. 12, $\mathrm{n}$ o 1, p. 97-117, [En ligne], http://chs.revues.org/70, consulté le 20 août 2015.

Halberstam, Judith, 2005, In a Queer Time and Place. Transgender Bodies, Subcultural Lives, New York, New York University Press.

Hamilton, Deborah, 1994, The French Detective Fiction Novel 1920s to 1990s: Gendering a Genre, thèse de doctorat inédite, Pennsylvania State University.

Hynynen, Andrea, 2013a, «Le roman policier et les trans' : une comparaison de Mygale par Thierry Jonquet et Transfixions par Brigitte Aubert », dans A. Alessandrin et al. (dir.), Corps Trans/ Corps Queer, Paris, L'Harmattan, p. 115-123.

-, 2013b, « Constructions du genre dans le roman policier "anti-norme” de Fred Vargas », Romanica Silesiana, $\mathrm{n}^{\circ}$ 8, p. 151-161. 
Kimyongür, Angela, 2013, « Dominique Manotti and the Roman Noir », Contemporary Women's Writing, vol. 7, $\mathrm{n}^{\circ}$ 3, p. 235-252.

Klein, Kathleen Gregory, 1988, The Women Detective. Gender \& Genre, Chicago, University of Illinois Press.

Mäntymäki, Tiina, 2004, Hard \& Soft. The Male Detective's Body in Contemporary European Crime Fiction , thèse de doctorat, Linköping, Linköping University Press.

Milestone, Katie et Meyer, Anneke, 2012, Gender and Popular Culture, Cambridge, Polity Press.

Munt, Sally, 1994, Murder by the Book? Feminism and the Crime Novel, Londres, Routledge.

Peach, Linden, 2006, Masquerade, Crime and Fiction. Criminal Deceptions, Basingstoke, Palgrave Macmillan.

Plain, Gill, 2001, Twentieth-Century Crime Fiction. Gender, Sexuality and the Body, Édimbourg, Edinburgh University Press.

Reddy, Maureen, 1988, Sisters in Crime: Feminism and the Crime Novel, New York, Continuum.

Richardson, Niall, 2010, Transgressive Bodies. Representations in Film and Popular Culture, Farnham, Ashgate.

Stewart, Faye, 2014, German Feminist Queer Crime Fiction. Politics, Justice and Desire, Jefferson, McFarland.

Sudret, Laurence, 2010, "Du traitement de la marginalité dans les "rompols" de Fred Vargas », dans G. Menegaldo et M. Petit (dir.), Manières de noir. La fiction policière contemporaine, Rennes, PUR, p. 221-236.

Tabachnik, Maud, 1997, « Remarques sur la non-place des femmes dans le roman noir », Les Temps modernes, nำ 595, p. 122-129.

Terry, Jennifer et Urla, Jacqueline (dir.), 1995, Deviant Bodies. Critical Perspectives on Difference in Science and Popular Culture, Bloomington, Indiana University Press.

Thomas, Maud-Yeuse et Espineira, Karine, 2015, «L'année 2014, année trans ? », Observatoire des transidentités, [En ligne], http://www.observatoire-des-transidentites.com/2015/01/1annee-2014-annee-trans.html, consulté le 30 janvier 2015.

Walton, Priscilla et Jones, Manina, 1999, Detective Agency. Women Rewriting the Hard-Boiled Tradition , Berkeley, University of California Press.

\section{Corpus de l'étude}

Aubert, Brigitte, [1998] 1999, Transfixions, Paris, Points policier. Première éd. : Seuil.

-, [2006] 2007, Une âme de trop, Paris, Points policier. Première éd. : Seuil.

Brac, Virginie, [2000] 2002, Tropique du pervers, Paris, Pocket. Première éd. : Fleuve noir.

-, [2002] 2003, Notre-Dame des barjots, Paris, Pocket. Première éd. : Fleuve noir.

-, [2004] 2005, Double peine, Paris, Pocket. Première éd. : Fleuve noir.

Lemaitre, Pierre, [2006] 2010, Travail soigné, Paris, Le Livre de poche. Première éd. : Éditions du Masque.

_, [2011] 2012, Alex, Paris, Le Livre de Poche. Première éd. : Albin Michel. 
-, 2012, Sacrifices, Paris, Albin Michel.

-, 2013, Rosy \& John, Paris, Le Livre de Poche.

Vargas, Fred, [2001] 2006, Pars vite et reviens tard, Paris, J’ai lu. Première éd. : Viviane Hamy.

-, [2004] 2008, Sous les vents de Neptune, Paris, J’ai lu. Première éd. : Viviane Hamy.

-, [2006] 2009, Dans les bois éternels, Paris, J’ai lu. Première éd. : Viviane Hamy.

-, 2008, Un lieu incertain, Paris, Viviane Hamy.

—, 2011, L'Armée furieuse, Paris, Viviane Hamy.

\section{NOTES}

1. Titre qui sera abrégé sous la forme de Une âme... pour les citations à venir dans le texte.

2. Virginie Brac, Tropique du pervers (2000); Notre-Dame des barjots (2002); Double peine (2004). Titres qui seront abrégés sous la forme de Tropique..., de Notre-Dame... et de Double peine pour les citations à venir dans le texte.

3. Pierre Lemaitre, Travail soigné (2006) ; Alex (2011) ; Sacrifices (2012). Titres qui seront abrégés sous la forme de Travail soigné, de Alex et de Sacrifices pour les citations à venir dans le texte.

4. Les romans étudiés sont (par date de parution originale) : Fred Vargas, Pars vite et reviens tard (2001); Sous les vents de Neptune (2004); Dans les bois éternels (2006); Un lieu incertain (2008); L'Armée furieuse (2011). Titres qui seront abrégés sous la forme de Pars vite..., de Sous les vents..., de Dans les bois..., de Un lieu... et de L'Armée furieuse pour les citations à venir dans le texte.

5. Nous utiliserons le mot " genre » pour parler du genre sexué (gender). Quand il est question de genre littéraire ou de genre grammatical, cela sera indiqué.

6. Deux livres pionniers ont été publiés en 1988: The Women Detective. Gender \& Genre écrit par Kathleen Gregory Klein et Sisters in Crime: Feminism and the Crime Novel de Maureen Reddy. Voir aussi Sally Munt (1994), et Priscilla Walton et Manina Jones (1999). Pour un aperçu des études gaies, lesbiennes et queer, voir Faye Stewart (2014).

7. Le livre Crimes suspects : femmes et hommes dans le roman policier de Danielle Charest (2006) est une des rares études francophones à ne pas se focaliser que sur la femme dans le roman policier. D'ailleurs, la plupart des recherches portant sur le féminisme ou les femmes dans le roman policier français sont publiées hors de France. Voir Deborah Hamilton (1994); Nicola Barfoot (2007) ; Véronique Desnain (2009) ; Angela Kimyongür (2013).

8. Sally Munt (1994 : 202) constate que les idées féministes se propageaient déjà dans le courant principal du roman policier anglophone au début des années 1990.

9. Plain $(2001: 3-5,244-248)$ écrit à forte raison que le rôle nodal occupé traditionnellement par le détective s'est peu à peu érodé. Même si nous trouvons des traces de cette évolution dans notre corpus, les détectives y figurant sont des figures héroïques et porte-parole des valeurs morales.

10. Voir « note de l'auteur».

11. Voir note 3.

12. Offert en cadeau pour l'achat de deux livres de poche, Rosy et John n'était pas accessible autrement et il est devenu quasiment introuvable.

13. Voir note 2 . 


\section{RÉSUMÉS}

L'article explore l'usage qui est fait de corps hors-norme par Brigitte Aubert, Virginie Brac, Pierre Lemaitre et Fred Vargas. Le corps trop petit de Verhœven, créé par Lemaitre, ainsi que celui, trop grand, de Retancourt, inventée par Vargas, ont une place notable dans les livres, mais la déviance corporelle ne touche que leur portrait; elle est peu importante pour le récit. Dans la série de Véra Cabral, créée par Brac, les allusions à son corps intersexe créent un deuxième mystère à côté de l'enquête criminelle. Aubert introduit dans Transfixions unE enquêteurE trans* en pleine transition, alors qu'Une âme de trop présente un protagoniste trans* qui est à la fois la victime et son propre assassin. La question qui se pose est celle de savoir si ces corps déstabilisent les normes de genre ou sont voués à les reproduire.

This article studies the deviant bodies of detectives in crime novels written by Brigitte Aubert, Virginie Brac, Pierre Lemaitre and Fred Vargas. The deviant body is either too big or too small according to gender expectations in Lemaitre's and Vargas's work, where its role is limited to characterization. The allusions to the intersex body of Brac's character Véra Cabral creates a secondary mystery alongside the criminal investigation. Aubert introduces a trans* investigator in transition in Transfixions, while the trans* protagonist is both murderer and victim in Une âme de trop. The study explores these bodies' function in the narratives, and how they relate to the plot. Furthermore, it asks whether they encourage the challenging of gender norms, or end up repeating them.

INDEX

Mots-clés : corps, déviance, norme, genre, transsexualité, intersexe

Keywords : body, norm, gender, transgender, intersex

\section{AUTEUR}

\section{ANDREA HYNYNEN}

University of Turku, Finlande, Turku Institute for Advanced Studies 